\title{
Global risk of cardiovascular disease
}

\section{N Poulter}

Heart 2003;89(Suppl II):ii2-ii5

UK death rates from coronary heart disease are among the highest in the world. This is because the UK has high levels of standard risk factors and a low level of intervention on those risk factors. The most important modifiable cardiovascular risk factors are dyslipidaemia (particularly high LDL cholesterol and low HDL cholesterol), smoking, hypertension, glucose intolerance, and central obesity. Intervention strategies that do not target those individuals at highest cardiovascular risk are likely to be less cost effective. Global risk estimation is increasingly recognised by management guidelines as a clinically and cost effective means of guiding treatment. However, an over reliance on short term absolute risk may result in under treatment of young people (particularly women) at high relative risk and over treatment of older people (particularly men) at low relative risk.

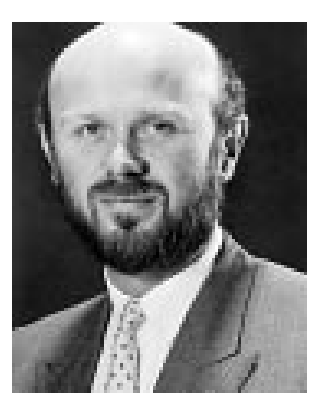

$\mathrm{D}$ espite understanding more about the aetiology and pathophysiology of cardiovascular disease and having more effective tools to prevent these diseases, the burden of cardiovascular disease is likely to worsen rather than improve over the next 20 years. In terms of global burden of disease, in 1999 the World Health Organization (WHO) placed ischaemic heart disease in sixth place and stroke in seventh place, but by 2020 they will have moved to first and fourth place, respectively. Total cardiovascular deaths from coronary heart disease (CHD), stroke, and other forms of cardiovascular disease are expected to almost double from 13.1 million in 1990 to 24.8 million in 2020. All cause mortality will also increase, reflecting the world's increasing population, but the projected increase $(35 \%)$ is small compared with the expected increase in cardiovascular deaths.

The explanation for this increase in cardiovascular disease is that most of the world is in the process of developing, and as populations develop their exposure to the standard risk factors for cardiovascular disease increases-for example, people get older, they smoke more, exercise less, drink more alcohol, their body weight increases, and intake of saturated fat and salt increases, while potassium intake decreases (because of lower consumption of fresh fruit and vegetables).

WHO data on global CHD mortality ( 1988 to 1991) show that the UK death rate from CHD is one of the highest in the world. The USA has achieved a pronounced reduction in CHD mortality over the past 30 years while former Eastern bloc countries, such as the Czech Republic, Slovakia, and Hungary, have seen a notable increase and currently have the highest CHD mortality rates. Huge population swings in disease morCardiovascular Studies Unit, Department of Clinical Pharmacology Imperial College London, $\mathrm{NHLl}$, Faculty of Medicine, W2 IPG, UK;

n.poulter@imperial.ac.uk. tality over a short time indicate that the disease cannot primarily be a genetic disorder. Indeed, CHD is primarily an environmental problem.

In the UK, CHD is the most common cause of premature death. It results in one in four male deaths and one in six female deaths, and caused around 125000 deaths in $2000 .^{1}$ There are around 274000 myocardial infarctions each year. CHD costs the National Health Service around $£ 1.6$ billion each year, only $1 \%$ of which is spent on primary prevention. The overall cost of CHD to the UK economy is around $£ 10$ billion each year.

\section{CARDIOVASCULAR RISK FACTORS}

The high UK cardiovascular death rate can be explained by the fact that there are high levels of standard risk factors and a low level of intervention on those risk factors. Over $300 \mathrm{CHD}$ risk factors have been cited in the literature. Table 1 lists those risk factors that are generally considered to be the most important. Non-modifiable risk factors are age, sex, family history, and, to a limited extent, genes. Birthweight is also included, in line with Barker's hypothesis.

There are 11 major modifiable risk factors, the most important of which is low density lipoprotein (LDL) cholesterol. A person with very low LDL cholesterol almost certainly will not have a myocardial infarction. The rural Japanese and Chinese have high rates of smoking but low rates of myocardial infarction and this is in part because they have very low concentrations of LDL cholesterol. That is commensurate with animal experimental data, which show the pivotal role of LDL cholesterol in producing atherosclerosis. In addition to dyslipidaemia, other key modifiable risk factors are smoking, hypertension, glucose intolerance, and central obesity ( the latter being a more reliable indicator of cardiovascular risk than body mass index).

The frequency distribution of LDL cholesterol is shifted to the right in patients with coronary artery disease but there is considerable overlap with controls. ${ }^{3}$ However, the importance of lipids at the population level can be clearly shown by comparing cholesterol distribution in Japan (which has low cholesterol and a very low incidence of myocardial infarction) and Finland (which has notably higher cholesterol concentrations and a very high incidence of myocardial infarction).

Protective factors in terms of CHD include moderate alcohol intake, exercise, dietary monounsaturated fats (largely obtained from olive

Professor Neil Poulter St Mary's Campus, London

Abbreviations: BHS, British Hypertension Society; $\mathrm{CHD}$, coronary heart disease; $\mathrm{HDL}$, high density lipoprotein; LDL, low density lipoprotein; LVH, left ventricular hypertrophy; NSF, National Service Framework; PROCAM, prospective cardiovascular Münster; WHO, World Health Organization 
Table 1 Risk factors for coronary heart disease

\begin{tabular}{ll}
\hline Modifiable & Non-modifiable \\
\hline High LDL cholesterol & Age \\
High blood pressure & Sex \\
Smoking & Family history \\
Low HDL cholesterol & Genetic \\
Lack of exercise & Birth weight \\
Diabetes and glucose intolerance & \\
Left ventricular hypertrophy & \\
Central obesity & \\
Homocysteine & \\
Clotting factors & \\
Oral contraceptives &
\end{tabular}

HDL, high density lipoprotein; LDL, low density lipoprotein. oil and rape seed oil, although red meat also has a high monounsaturated fat content), fruit and vegetables, high HDL cholesterol, fish, and, at least in secondary prevention and some primary prevention, aspirin. The mortality rate for CHD is much higher in men than women. People have concluded from this that oestrogen is protective in some way, although there is growing evidence to suggest that this is not the case. $^{45}$

An individual's risk of CHD can only be determined by taking account of all of these different risk factors and protective factors.

\section{RISK STRATIFICATION}

It is important to appreciate that the various risk factors for CHD interact. Historically, individual risk factors have been managed in isolation of other concomitant problems. Hugh Tunstall-Pedoe commented over 10 years ago: "Coronary risk is multifactorial, but most medical algorithms for risk factor management, while paying lip service to the others, consider them one at a time." ${ }^{6}$ Thus, different specialistsdiabetologists, lipidologists, blood pressure specialists-have traditionally focused on individual risk factors. For example, a patient with a fasting glucose greater than $7 \mathrm{mmol} / \mathrm{l}$ was diagnosed as diabetic and treated, and a patient with systolic blood pressure repeatedly greater than $160 \mathrm{~mm} \mathrm{Hg}$ was diagnosed as hypertensive and treated. The concept of "global risk"taking account of all risk factors to estimate an individual's absolute risk of CHD —was introduced to guide treatment of patients with hyperlipidaemia, but it is equally important in diabetes and hypertension.

However, progress has been made and the most recent US Joint National Committee report on hypertension management introduced a crude classification of risk. ${ }^{7}$ It defines three levels of blood pressure and three groupings of risk factors: no risk factors and no target organ damage; one risk factor (not diabetes) and no target organ damage; and a higher risk group with target organ damage or CHD. Depending on the risk group and the level of blood pressure, lifestyle modification or drug treatment plus lifestyle modification is recommended.

The idea of basing management on risk stratification continued with the British Hypertension Society (BHS) 1999 guidelines for management of hypertension, which introduced thresholds for intervention. ${ }^{8}$ Essentially, these guidelines recommend treatment if the blood pressure is $\geqslant 160 /$ $100 \mathrm{~mm} \mathrm{Hg}$, no treatment if it is $<140 / 90 \mathrm{~mm} \mathrm{Hg}$, and examination of risk factor status if it is in the "grey area" of 140-159/90-99 mm Hg. In this third group, treatment is recommended if the individual has target organ damage, cardiovascular complications or diabetes, or if their estimated 10 year CHD risk is $\geqslant 15 \%$. The BHS guidelines therefore introduced a global risk estimate of when to treat for a large proportion of the population.
A global risk estimate is also included in the National Service Framework (NSF) for CHD, ${ }^{9}$ which recommends lipid lowering treatment with statins if patients have a greater than $30 \%$ chance of a coronary event over the next 10 years. There is, in fact, evidence showing benefits with lipid lowering at $6 \%$ risk, one fifth of the level at which the NSF recommends treatment, and this recommendation has therefore little to do with science and is purely based on financial considerations.

The BHS guidelines also advise on when to give low dose aspirin according to risk: the drug is recommended for all patients requiring secondary prevention and in primary prevention above a certain level of risk, defined here as patients aged 50 years and over who have satisfactory control of their blood pressure and target organ damage, diabetes or a 10 year $\mathrm{CHD}$ risk of $\geqslant 15 \% .{ }^{8}$

\section{HOW TO CALCULATE AN INDIVIDUAL'S RISK}

Intervention strategies that do not target patients at highest cardiovascular risk are likely to be less cost effective than those that do. There are several different systems for evaluating an individual's risk of CHD. The Dundee risk $\operatorname{disc}^{6}$ is a simple system in principle; it can, however, be tricky to use. The American Heart Association system is based on data from the Framingham study. This small cohort study (5209 subjects) also forms the basis for the Joint British Societies' coronary risk prediction chart ${ }^{10}$ and for risk charts used in several other countries. A further system is based on data from the British regional heart study, ${ }_{11}$ while another system has been based on the German PROCAM (prospective cardiovascular Münster) cohort. ${ }^{12}$

Each of these risk assessment systems includes different variables. They also differ in how they quantify the standard variables, such as smoking. The Dundee risk disc differentiates between never-smoker, ex-smoker (quantified in terms of years since stopping), pipes and cigars, and the average number of cigarettes per day. ${ }^{6}$ The British regional heart study risk function uses "years smoked", which was found to be the best predictor in that database. ${ }^{11}$ The systems based on Framingham data, and on the PROCAM data, simply have a yes/no answer, which means that a person smoking one cigarette a day is classed as a smoker while someone who stopped a year ago having smoked 60 cigarettes a day for 40 years is classed as a non-smoker. That clearly is an important problem. This demonstrates that the data entered into these scores are crude, and so can only lead to crude risk estimates.

Framingham risk factor scoring calculates a 10 year estimated risk of CHD based on points allocated for age, total cholesterol, smoking status, high density lipoprotein (HDL) cholesterol, and systolic blood pressure. ${ }^{13}$ Most of the risk assessment systems that are currently widely used are based on the Framingham data. There has been debate as to whether it is reasonable to extrapolate data collected from a white middle class population in Massachusetts to other parts of the world. However, a study from Sheffield ${ }^{14}$ found little difference between the line of identity and the regression line between Framingham data and the northern European PROCAM database (fig 1).

Using the Framingham score, the Joint British Societies produced two systems for calculating absolute risk of CHD: the cardiac risk assessor computer program and the CHD risk chart. ${ }^{10}$ It could be argued that we should be assessing cardiovascular disease risk rather than CHD risk, as it is important to know an individual's risk of stroke as well as of myocardial infarction. However, when the Joint British Societies' systems were being prepared, other systems that assessed CHD risk were already available and it was thought that cardiovascular disease risk charts might cause confusion. The CHD risk can be converted to give a rough estimate of cardiovascular disease risk by multiplying by $4 / 3$ (for example, a $30 \%$ CHD risk is 


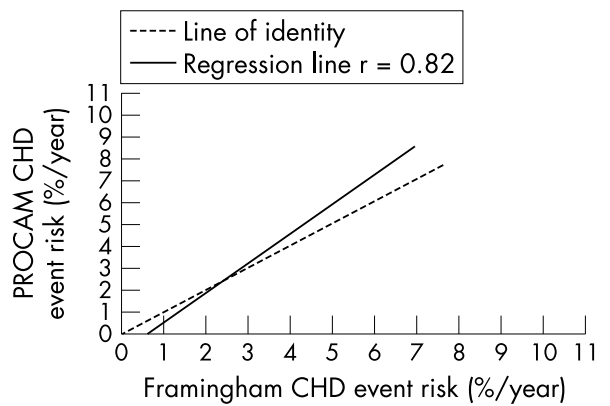

Figure 1 Does the Framingham risk function accurately predict $\mathrm{CHD}$ risk in a European population? Adapted from Haq et al, ${ }^{14}$ with permission.

equivalent to a $40 \%$ cardiovascular disease risk), although the conversion is less accurate at the extremes of age.

The Joint British Societies' risk chart takes account of sex, age, smoking status, systolic blood pressure, and total:HDL cholesterol ratio. This gives a guide to the individual's level of CHD risk, dividing it into three groups:

- > 30\% estimated risk over the next 10 years

- $15-30 \%$ risk over the next 10 years

- $<15 \%$ risk over the next 10 years.

Separate charts are produced for non-diabetic and diabetic subjects, although it might be argued that a patient with diabetes should automatically attract treatment as for secondary prevention and that risk does not need to be calculated.

The chart is relatively simple to use and is the most accurate of all the available risk charts. There are, however, a few inherent problems. One of these is that the use of short term absolute risk as a determinant of intervention causes under treatment of young people at high relative risk and over treatment of older people at low relative risk. For example, a 32 year old woman, even if she is diabetic, a smoker, has a total cholesterol:HDL ratio of 10, and a systolic blood pressure of $180 \mathrm{~mm} \mathrm{Hg}$, does not reach the 30\% risk level at which intervention is recommended. In contrast, most elderly men would qualify for intervention simply on account of their age and sex. The European approach is to "project" young people with high levels of risk factors to age 60 and to base treatment decisions on the resulting estimated level of risk, which is one way of avoiding the ageism and sexism inherent in these charts.

The computerised Joint British Societies' risk factor assessment is based on the following variables: sex, age, systolic blood pressure, diastolic blood pressure, smoker (yes/no), serum cholesterol, HDL cholesterol, diabetes (yes/no), and left ventricular hypertrophy on ECG (yes/no). There are limitations with this scoring system. For example, as for smoking, the scoring system for diabetes is based on a yes/no answer rather than on the $\mathrm{HbA}_{\mathrm{IC}}$ concentration, and so it does not reflect the fact that risk is graded across the scale for glucose concentrations, as it is for smoking habit. However, the computerised assessment is more accurate than the charts, it is easy to use, and it has helped doctors in their interaction with patients.

There are seven ideal requirements of a risk factor scoring system:

- simple

- comprehensive

- cheap

- valid and predictive (which means it should be based on the population to which it is going to be applied, which is very difficult)

\section{Learning points}

- Coronary heart disease is not primarily a genetic disorder

- Worldwide, cardiovascular disease is going to get worse, particularly in developing countries. It is essential that appropriate strategies are developed to deal with this

- The absolute risk score-global risk score-is appropriate and should be applied to diabetes and blood pressure, not just to lipids. But it is necessary to be aware that concentrating on short term absolute risk will divert resources to old men at the expense of "at risk" young people

- user friendly

- easily interpreted by the doctor and the patient

- useful (to identify those at high risk, to motivate patients and doctors, to monitor their well-being as a result of interventions, and to improve management).

It will be difficult for one scoring system to meet all those requirements, and methods of evaluating absolute risk, if they are to be widely used, involve a trade-off between accuracy and simplicity. But the absolute risk score-a global risk score-is a really useful tool to guide risk management, as long as its shortcomings are recognised.

\section{CONCLUSION}

Intervention strategies that do not target those at highest cardiovascular risk are likely to be less cost effective. Global risk estimation, taking account of all risk factors to produce an assessment of an individual's absolute risk of CHD, is increasingly recognised by management guidelines as a clinically and cost effective means of guiding treatment. However, the limitations of using short term absolute risk to determine intervention need to be recognised.

\section{REFERENCES}

1 British Heart Foundation Database. Coronary heart disease statistics, 2002 edition. London: British Heart Foundation. URL: www.dphpc.ox.ac.uk/bhfhprg/stats/2000/2002/keyfacts/index.html (accessed 12 February 2003).

2 Barker DJP. Fetal origins of coronary heart disease. BM $1995 ; 311: 171-4$

3 Barbir M, Wile D, Trayner I, et al. High prevalence of hypertriglyceridaemia and apolipoprotein abnormalities in coronary artery disease. Br Heart J 1988;60:397-403.

4 Poulter N. Oestrogen and protection against cardiovascular disease: potential versus reality. Br J Cardiol 2001;8:383-90.

5 Beral V, Banks $E$, Reeves $G$. Evidence from randomised trials on the long-term effects of hormone replacement therapy. Lancet 2002;360:942-4

6 Tunstall-Pedoe $\mathbf{H}$. The Dundee coronary risk-disk for management of change in risk factors. BM 1991;303:744-7

7 Joint National Committee. The sixth report of the Joint National Committee on prevention, detection, evaluation and treatment of high blood pressure. Arch Int Med 1997;157:2413-46.

8 Ramsay LE, Williams B, Johnston GD, et al, for the British Hypertension Society. Guidelines for management of hypertension: report of the third working party of the British Hypertension Society. J Hum Hypertens 1999; 13:569-92.

9 Department of Health. National service framework for coronary heart disease. London: Department of Health, 2000.

10 British Cardiac Society, British Hyperlipidaemia Association, British Hypertension Society, British Diabetic Association. Joint British recommendations on prevention of coronary heart disease in clinical practice. Heart 1998;80(suppl 2):S1-29.

11 Shaper AG, Pocock SJ, Phillips AN, et al. Identifying men at high risk of heart attacks: strategy for use in general practice. BM 1986;293:474-9

12 Assmann G, Cullen P, Schulte H. Simple scoring scheme for calculating the risk of acute coronary events based on the 10-year follow-up of the prospective cardiovascular Munster (PROCAM) study. Circulation 2002; 105:310-5

13 National Cholesterol Education Program. Executive summary of the third report of the National Cholesterol Education Program (NCEP) expert panel on detection, evaluation, and treatment of high blood cholesterol in adults (adult treatment panel III). JAMA 2001;285:2486-97.

14 Haq IU, Ramsay LE, Yeo WW, et al. Is the Framingham risk function valid for northern European populations? A comparison of methods for estimating absolute coronary risk in high risk men. Heart 1999;81:40-6. 


\section{DISCUSSION}

Professor Ritchie: Is coronary risk passé and cardiovascular risk the way forward?

Professor Poulter: The revised Joint British Societies' guidelines that will be coming out next year are going to move towards cardiovascular risk, and I hope that all the other guidelines will make a similar move.

Question: How do we interpret left ventricular hypertrophy [LVH] that has arisen in an athletic youth in terms of coronary risk?

Professor Poulter: No-one really knows the answer to this. It can be associated with sudden death, but in terms of risk it is clearly different from the LVH that has come about through hypertension. There is currently no way of dealing with this in risk assessment.

Comment: I would like to defend the Framingham study. Although it is a relatively small cohort in present terms, when set up in the 1950s it was probably seen as quite large. And it has certainly helped us in terms of the power to predict important risk factors.

Professor Poulter: I thoroughly agree with you. I don't think there is much wrong with Framingham.

Comment: I think your point of trying to quantify risk in a useful way is very important. Most of the population at large in the UK understands very little about risk.

Professor Poulter: Since UK doctors have been arguing about cholesterol until the last few years, it is difficult to expect the population to know what is going on. We are still arguing about salt (although the rest of the world has accepted this) and until the medical profession can reach agreement the general population has got little chance. Even if we start agreeing what the risk factors are, there are difficulties in getting the message across. Do you tell a patient about relative risk, for example, that they are five times more likely to have a heart attack than someone else of their age, or do you say they have a 1 in 100000 chance?

Question: Why are the CHD figures so poor in the UK relative to France and the USA where people have apparently less healthy lifestyles? Is it because of earlier use of statins and aspirin in those countries?

Professor Poulter: They do use more statins and aspirin, but the perception that the French have got a less healthy lifestyle than us is wrong. Their total fat intake is about the same as ours but their diet includes much more monounsaturated fat. They eat three times the amount of fresh fruit and vegetables than people in Scotland. Remember too that we are talking about a chronic degenerative disease. It can be misleading to try to relate current levels of risk factors to current levels of coronary events. You have got to go back 40 or 50 years at which point smoking in 20 year olds was much more common in Britain than in France. So, we are now seeing the impact of what happened in the 1950s. The American risk factor status is very much better than ours. 